\title{
Quantization noise: An additional constraint for the extended sampling theorem.
}

\author{
Damien P. Kelly* ${ }^{1}$, Nitesh Pandey ${ }^{1}$, Bryan M. Hennelly ${ }^{1}$ and Thomas J. Naughton ${ }^{1,2}$ \\ ${ }^{1}$ Department of Computer Science \\ National University of Ireland, \\ Maynooth, Co. Kildare, Ireland. \\ ${ }^{2}$ University of Oulu, RFMedia Laboratory, \\ Oulu Southern Institute, Vierimaantie 5, 84100 Ylivieska, \\ Finland. \\ *damienk@cs.nuim.ie
}

Abstract: Recovery of spatial frequencies above the Nyquist limit is of interest in digital holography. We examine how finite pixel size and quantization error introduced by a CCD camera effect the recovery of these frequencies.

(C2009 Optical Society of America.

OCIS codes: (090.1995) Digital Holography; (070.2025) Discrete optical signal processing; (100.6640)

Superresolution

\section{Introduction}

Digital holography (DH) differs from traditional holography in that a dynamic digital sensor is used in place of photographic plate to record the interference pattern due to an incident object and reference wave-field. By recording the interference pattern of an object and reference wave the complex amplitude of the object field may be recovered [1-6]. There are many advantages to a digital approach such as ease of use, flexibility in image processing, storing and transmission of the data electronically. However the limited number of pixels on a modern CCD camera (as well as the relatively large spacing between adjacent pixels in contrast to a comparable recording on typical photographic film) imposes a severe limitation on the spatial resolution of the captured digital holograms. High spatial resolution in digital holograms is important for accurately recording and replaying $3 \mathrm{D}$ scenes $[7,8]$.

Let us assume that the field in the object plane, $u(X)$, contains a maximum spatial frequency $f_{\mathrm{x}}$. This field now propagates, which we describe under paraxial conditions using the Fresnel transform, to the camera place where its complex amplitude, $u_{\mathrm{z}}(x)$, is recorded. Since the magnitude of the spatial frequency content of our signal remains unchanged under Fresnel propagation, the field at the camera plane must also contain the spatial frequency $f_{\mathrm{x}}$. Our recording CCD camera has some spatial frequency bandwidth, $B_{\mathrm{c}}=2 f_{\mathrm{c}}$ (Nyquist rate), that is determined by the spacing between adjacent pixels on the camera face. On initial examination one might suppose that $f_{\mathrm{c}} \geq f_{\mathrm{x}}$ if the camera is to be able to resolve $f_{\mathrm{x}}$, however many authors have shown that this Nyquist criterion may be too strict [915] and that it is possible to recover $f_{\mathrm{x}}$ under certain conditions. Since this has been derived elsewhere we shall merely state the result here: A signal $u(X)$ that spans a finite extent, $\Delta$, may be fully recovered provided that its entire Fresnel transform, $u_{\mathrm{z}}(x)$, is sampled at a rate $\delta$, such that

$$
\delta \leq \Delta /(\lambda z)
$$

where $\lambda$ is the wavelength of the light and $z$ the perpendicular distance between object and camera (capture) planes.

In the derivation of Eq. (1) it has been assumed that the field, $u_{\mathrm{z}}(x)$, is sampled at the camera face at infinitely narrow and discrete points which can be modelled using Dirac delta functions [13]. Physically however the camera pixels extend over a finite space and average the light energy incident upon them. This averaging process is mathematically equivalent to multiplying those spatial frequencies contained in the object wave-field by a $\sin (x) / x$ type function with the result that higher spatial frequencies are attenuated relative to lower spatial frequencies [10-11,16-17]. We depict this schematically in Fig. 1(a). Therefore in addition to the previous sampling guideline, [see Eq. (1)], it appears from Fig. 1(a) that recovery of higher spatial frequencies will ultimately be limited by system noise present in all practical DH systems [10]. In this manuscript we examine a fundamental noise limit - quantization error - introduced by the finite accuracy with which an $N$-bit CCD pixel can 


\section{DWB12.pdf}

represent a number. Spatial frequencies that have been attenuated to a level below this minimum quantization level cannot be recovered, imposing an additional and fundamental constraint on the recovery of "super-Nyquist" frequencies.
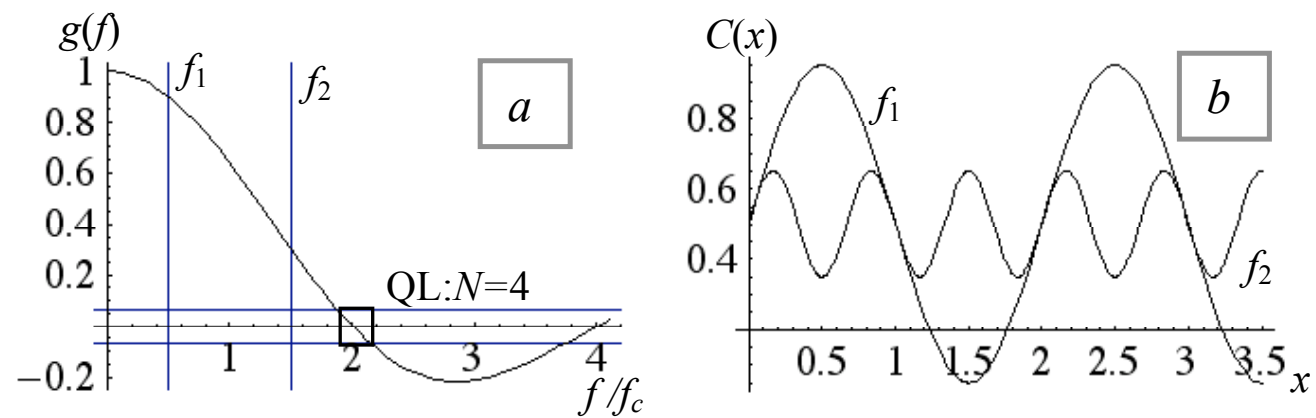

Fig 1 (a) Plot of $g(f)$ as a function of $f / f_{\mathrm{c}}$ where $f_{\mathrm{c}}$ is the Nyquist limit, QL - quantization limit, (b)

Analysis

the resulting contrast of the sin waves $f_{1}$ and $f_{2}$.

Let us now consider the interference equation for in-line digital holography when the finite size of the pixel, $\varsigma$, is taken into consideration. This equation is of the form [10]

$$
\begin{gathered}
I=I_{\mathrm{R}}+I_{\mathrm{O}}+2 A_{\mathrm{R}} A_{\mathrm{O}} \cos \left(\phi_{\mathrm{R}}-\phi_{\mathrm{O}}\right) g(f), \\
g(f)=\frac{\sin [2 \pi f(\varsigma / 2)]}{2 \pi f(\varsigma / 2)}
\end{gathered}
$$

where $I, A$, and $\phi$ indicate intensity, amplitude and phase respectively and where the subscripts $\mathrm{R}$ and $\mathrm{O}$ refer to the reference and object wave-fields respectively. Eq. $(2 \mathrm{~b})$ is a spatial frequency dependent weighting that arises due to the finite extent of the camera pixel and is plotted in Fig. 1(a). With no loss in generality we may consider a simple form for the object and reference fields namely:

$$
\begin{aligned}
& A_{\mathrm{R}}=K_{1} \exp (j \alpha) \\
& A_{\mathrm{O}}=K_{2} \exp (j 2 \pi f x)
\end{aligned}
$$

where $f$ is known as the spatial frequency of an incident plane wave and can be related to the physical wavelength of the light, $\lambda$, and $\theta$ the angle made with respect to the $z$-axis by the relation $f=$ $\sin (\theta) / \lambda$ [2]. Note that the highest non-evanescent spatial frequency occurs when $\theta=\pi / 2$ and thus $f_{\max }=1 / \lambda$. We choose values for $K_{1}$ and $K_{2}$ such that the resulting difference between the maximum and minimum value [which we refer to as contrast $C(x)$ ] in Eq. $\left(2\right.$ a) is unity when $f=0$, i.e. $2 K_{1} K_{2}=$ 0.5. In Fig. 1(b) we show how two different spatial frequencies, $f_{1}$ and $f_{2}$ [see Fig. 1(a)], are recorded by the camera and note that the higher spatial frequency $f_{2}$ is attenuated relative to $f_{1}$. Indeed as the spatial frequency increases the contrast of the resulting interference fringe decreases according to $g(f)$, until eventually the spatial frequency amplitude is less than the minimum quantization level that can be represented using an $N$ bit word. We now examine when this quantization limit is reached. We adjust the dynamic range of the camera so that it spans zero to unity and assume that the $N$ bits available to represent values within this range are divided into uniform step sizes. From Ref. [18] the minimum step size is given by

$$
\Delta I=\frac{2\left(2 K_{1} K_{2}\right)}{2^{N}-1}=\frac{1}{2^{N}-1} .
$$

It is not be possible to measure spatial frequencies when the maximum interference fringe amplitude is less than this minimum size, $\Delta I$. Examining Eq. $(2 \mathrm{~b})$ we see that when $f_{n}=(2 n+0.5) / \varsigma$, $\sin [2 \pi f(\varsigma / 2)] \rightarrow 1$ and $g\left(f_{n}\right)=\pi^{-1}(0.5+2 n)^{-1}$, where $n$ is a natural number greater than zero. We now use this latter expression to determine when the spatial frequencies have dropped below the critical quantization level $\Delta I$, by equating it with Eq. (4). Doing so we arrive at the equations

$$
n \approx-0.409+0.1592^{N} \text {, }
$$




\section{DWB12.pdf}

$$
f_{n} \approx 0.318\left(2^{N}-1\right) / \varsigma
$$

\section{Conclusion}

In this manuscript we have examined a fundamental limit on the recovery of spatial frequencies imposed by a combination of the finite size of the pixels in a CCD camera and the quantization error that occurs when using a finite number of levels $N$, to represent a number. From the result in Eq. (5 b) we see that the maximum recoverable frequency is dependent on both the bit depth $N$ and the pixel width $\varsigma$. When $N$ is small $N \leq 4$ bits then Eq. $(5 \mathrm{~b})$ does indeed act to limit the range of spatial frequencies recoverable. For example for a value of $\varsigma=7.4 \times 10^{-6}$, then when $N=4, f_{\mathrm{n}} \sim 9.5 f_{\mathrm{c}}$. However, for larger bit rates, i.e. $N=10, f_{\mathrm{n}} \sim 162 f_{\mathrm{c}}$, (where $f_{\mathrm{c}}$ is the Nyquist rate), the bit rate may not in fact be the limiting factor for the recovery of physical spatial frequencies. For example with a wavelength of $\lambda=633 \mathrm{~nm}$, the maximum physical spatial frequency is given by $f_{\max }=1 / \lambda$ which is lower than the $162 f_{\mathrm{c}}$ predicted by Eq. $(5 \mathrm{~b})$.

These considerations must be further qualified. For a given distribution it is unlikely that all spatial frequencies will have the same magnitude - there will be variations in the magnitudes of the different spatial frequency components. Often for real world distributions, higher spatial frequencies contribute less energy to image formation. Ideally a statistical knowledge of likely spatial frequencies distributions for certain image types could be used in conjunction with Eq. $(5 \mathrm{~b})$ to determine the effect of quantization error. It is also important to note that the quantization effect will affect recovery of certain spatial frequencies below the limit identified in Eq. (5 b). This is depicted in Fig 1(a) by a black box around $f / f_{\mathrm{c}}=2$ for a value of $N=4$. We also note that as is discussed in Ref. [18] quantization will also introduce a bit dependent phase error in the argument of Eq. (2 a) - see Fig. 2 and 3 therein.

\section{Acknowledgements}

The research leading to these results has received funding from the European Community's Seventh Framework Programme FP7/2007-2013 under grant agreement no. 216105.

\section{References}

[1] D. Gabor, “A new microscope principle," Nature 161, 777-778 (1948).

[2] J. Goodman, Introduction to Fourier Optics, 2nd ed., McGraw-Hill, New York, (1966).

[3] M. Testorf and A. W. Lohmann, "Holography in phase space," Appl. Opt. A 47, 70-77 (2008).

[4] J. W. Goodman and R. Lawrence, "Digital image formation from electronically detected holograms," Appl. Phys. Lett. 11, 77-79 (1967).

[5] U. Schnars and W. Juptner, "Digital recording and numerical reconstruction of holograms," Meas. Sci. Technol. 13, 85-101 (2002).

[6] I. Yamaguchi and T. Zhang, "Phase-shifting digital holography," Opt. Lett. 22, 1268-1270 (1997).

[7] U. Gopinathan, D. S. Monaghan, B. M. Hennelly, C. P. M. Elhinney, D. P. Kelly, J. McDonald, T. J. Naughton, and J. T. Sheridan, "A projection system for real world three-dimensional ob jects using spatial light modulators," J. Display Technol. 4, 254-261 (2008).

[8] A. Michalkiewicz, M. Kujawinska, J. Krezel, L. Salbut, X. Wang, and P. J. Bos, "Phase manipulation and optoelectronic reconstruction of digital holograms by means of lcos spatial light modulator," Eighth International Symposium on Laser Metrology SPIE 5776(1) 144-152, , (2005).

[9] A. Stern and B. Javidi, "Improved-resolution digital holography using the generalized sampling theorem for locally bandlimited fields," J. Opt. Soc. Am. A23, 1227-1235 (2006).

[10] D. P. Kelly, B. M. Hennelly, C. McElhinney, and T. J. Naughton, “,A Practical Guide to Digital Holography and Generalized Sampling" 7072, 707215 (2008)

[11] D. P. Kelly, B. M. Hennelly, C. McElhinney, and T. J. Naughton, “A practical model for sub-Nyquist sampling in digital holography," submitted to Opt. Eng 10 Dec (2008).

[12] F. Gori, "Fresnel transform and sampling theorem," Opt. Commun. 39, 293-297 (1981).

[13] L. Onural, "Sampling of the Diffraction Field," Appl. Opt. 39, 5929-5935 (2000).

[14] A. Stern and B. Javidi, "Sampling in the light of Wigner distribution," J. Opt. Soc. Am. A 21, 360-366 (2004).

[15] A. Stern and B. Javidi, "Sampling in the light of Wigner distribution: errata," J. Opt. Soc. Am. A 21, 2038-2038 (2004).

[16] T. M. Kreis, "Frequency analysis of digital holography,"Opt. Eng. 41, 771-778 (2002).

[17] H. Jin, H. Wan, Y. Zhang, Y. Li, and P. Qiu, J. Mod. Opt. 55, 2989-3000 (2008).

[18] O. Skydan, F. Lilley, M. Lalor, and D. Burton, "Quantization Error of CCD Cameras and Their Influence on Phase Calculation in Fringe Pattern Analysis," Appl. Opt. 42, 5302-5307 (2003). 